\title{
On the distribution of the distances of multiples of an irrational number to the nearest integer
}

by

\author{
Henk Don (Delft)
}

1. Introduction. Take an arbitrary irrational number $\alpha$ and compute for the first $n$ multiples the distance to the nearest integer. What can we say about the distribution of this sequence in the interval $[0,1 / 2]$ ? In this paper we study the partition of the interval $[0,1 / 2]$ induced by this sequence. The main result (Theorem 2) states that this sequence divides the interval into subintervals which can take at most four different lengths. This result is strongly related to the Three Gap Theorem, which states that for $\alpha$ irrational and $n \in \mathbb{N}$, the numbers

$$
\{\alpha\},\{2 \alpha\},\{3 \alpha\}, \ldots,\{n \alpha\}
$$

divide the interval $[0,1]$ into subintervals of at most three different lengths. Here $\{x\}=x-\lfloor x\rfloor=x \bmod 1$ is the fractional part of $x$. The Three Gap Theorem was originally a conjecture of H. Steinhaus. Proofs were offered by various authors, for example by Sós [3], Świerczkowski [5], Surányi [4], Slater [2] and van Ravenstein [1].

We start with Theorem 1, a variation on the Three Gap Theorem, which states that if we divide the interval $[0,1]$ into subintervals by the numbers

$$
\{\alpha\},\{-\alpha\},\{2 \alpha\},\{-2 \alpha\}, \ldots,\{n \alpha\},\{-n \alpha\}
$$

then the subintervals again have at most three different lengths. We give an elementary proof for this theorem.

From Theorem 1 we extract the main result, Theorem 2. This "Four Gap Theorem" gives an analogous statement about the distances to the nearest integers of the multiples of $\alpha$ : the numbers

$$
\|\alpha\|,\|2 \alpha\|,\|3 \alpha\|, \ldots,\|n \alpha\|
$$

divide the interval $[0,1 / 2]$ into subintervals of at least two and at most

2010 Mathematics Subject Classification: Primary 11K06; Secondary 11B83, 11J71.

Key words and phrases: Steinhaus theorem, Three Gap Theorem, distance to nearest integer. 
four different lengths, where $\|x\|$ denotes the distance from $x$ to the nearest integer. Here the number four is the best possible. We also derive some properties of the lengths of the subintervals into which $[0,1 / 2]$ is divided.

2. A variation on the Three Gap Theorem. If we consider not only the fractional parts of the positive multiples of an irrational number $\alpha$, but also of the negative multiples, we have the following result:

THEOREM 1. Let $\alpha$ be an irrational number between 0 and 1 , and let $n \in \mathbb{N}, n \geq 1$. For the first $n$ numbers in the sequence

$$
S_{\alpha}:\{\alpha\},\{-\alpha\},\{2 \alpha\},\{-2 \alpha\},\{3 \alpha\},\{-3 \alpha\}, \ldots
$$

the following assertions hold:

1. They divide the interval $[0,1]$ into subintervals of either two or three different lengths, $l_{1}>l_{2}\left(>l_{3}\right)$. If we have three different lengths, $l_{1}>l_{2}>l_{3}$, then $l_{1}=l_{2}+l_{3}$.

2. By adding the $(n+1)$ th element of the sequence $S_{\alpha}$ to the partition of $[0,1]$, one of the subintervals of length $l_{1}$ is divided into a subinterval of length $l_{2}$ and a subinterval of length $l_{1}-l_{2}$.

Before proving the assertions we make some preparations by collecting observations that will be helpful in proving the assertions. Note that it makes no difference in Theorem 1 if we consider the open interval $(0,1)$.

First note that for $x \in \mathbb{R} \backslash \mathbb{Z}$ we have $\{-x\}=1-\{x\}$, so the partition induced by the first $2 n$ terms of the sequence $S_{\alpha}$ is symmetric with respect to $1 / 2$. This also means that without loss of generality we may assume that $\alpha<1 / 2$. Sometimes $\alpha$ will be called the step size.

It will prove useful to introduce some notation and definitions. For $n \geq 1$, $S_{\alpha}(n)$ denotes the $n$th term of $S_{\alpha}$. For each $k \in \mathbb{N}, k \geq 1$, let $n_{k}(\alpha)$ be the unique integer for which

$$
n_{k}(\alpha) \alpha<k<\left(n_{k}(\alpha)+1\right) \alpha .
$$

Since $\alpha$ is irrational, $k$ can never be a multiple of $\alpha$. Define $\beta$ by

$$
\beta:=\left(n_{1}(\alpha)+1\right) \alpha-1 \text {. }
$$

Note that $\beta=\left\{\left(n_{1}(\alpha)+1\right) \alpha\right\}$. Figure 1 illustrates these definitions in case $n_{1}(\alpha)=3$.

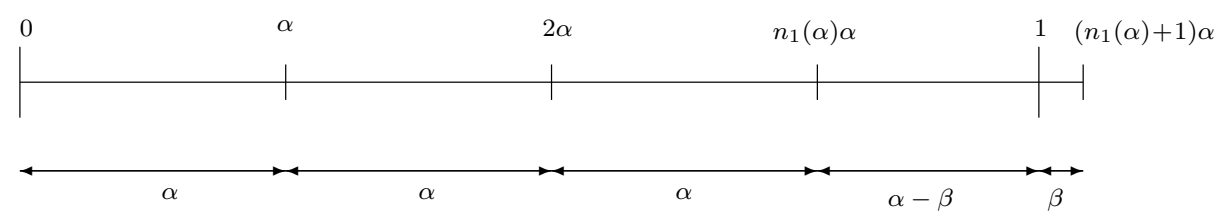

Fig. 1. The first steps in the partition process 
Definition 1 . For $k \in \mathbb{N}, k \geq 1$, the $k$ th cycle of the sequence $S_{\alpha}$ consists of all those fractional parts $\{m \alpha\}, m \in \mathbb{Z}$, for which $k-1<|m \alpha|<k$, or equivalently $n_{k-1}(\alpha)<|m| \leq n_{k}(\alpha)$.

Observe that a cycle consists of at least four partition points, because we assumed that $\alpha<1 / 2$. We are going to use this observation later. The next definition concerns intervals which are partitioned in the same way.

Definition 2. For $0 \leq a, b<1, y \leq \min \{1-a, 1-b\}$ and $n \in \mathbb{N}$ we write $(a, a+y)(n) \simeq(b, b+y)(n)$ if for all $x \in(0, y)$ the condition

$$
\exists k_{1} \in \mathbb{Z},\left|k_{1}\right| \leq n \text { such that } a+x=\left\{k_{1} \alpha\right\}
$$

is equivalent to

$$
\exists k_{2} \in \mathbb{Z},\left|k_{2}\right| \leq n \text { such that } b+x=\left\{k_{2} \alpha\right\} .
$$

Note that $\simeq$ is an equivalence relation on the class of partitioned open subintervals of $(0,1)$. If we replace $b+x$ by $b+y-x$ in Definition 2 , we get an equivalence for an interval and the mirror image of another interval. If two intervals satisfy this adjusted definition, we will write $(a, a+y)(n) \stackrel{m}{\simeq}$ $(b, b+y)(n)$.

Now let us investigate what happens in the interval $(0, \alpha)$. Note that $S_{\alpha}$ is a sequence in the open interval $(0,1)$. Therefore also here we investigate which values we get in the open interval $(0, \alpha)$. For $k \in \mathbb{N}, k \geq 1$, the interval $(k, k+\alpha)$ contains exactly one positive multiple of $\alpha$ and the interval $(-k,-k+\alpha)$ contains exactly one negative multiple of $\alpha$. Hence, in each cycle we get two values in $(0, \alpha)$, one of them being the fractional part of a positive multiple of $\alpha$ and the other being the fractional part of a negative multiple of $\alpha$. The first cycle is the only exception, since there is no positive multiple of $\alpha$ in $(0, \alpha)$.

The first positive multiple of $\alpha$ for which the fractional part is in $(0, \alpha)$ is $\left(n_{1}(\alpha)+1\right) \alpha=1+\beta$, which gives $\beta$ as a first hit in $(0, \alpha)$. Because $1+\beta$ is a positive multiple of $\alpha$, for $k \in \mathbb{N}$ the number $k+k \beta$ is also a positive multiple of $\alpha$. The fractional parts of these numbers are fractional parts of multiples of $\beta$. As long as $k \beta<\alpha$ this gives hits in $(0, \alpha)$. As soon as $k \beta$ exceeds $\alpha$, i.e. when $k=\lfloor\alpha / \beta\rfloor+1$, we leave the interval $(0, \alpha)$, but in that case we had already hit the value $k \beta-\alpha$. This is exactly how it continues all the time: each next hit in $(0, \alpha)$ is shifted $\beta$ in the positive direction, and as soon as we leave the interval, we come back modulo $\alpha$. Hence, for every positive integer $k$, the $k$ th hit in $(0, \alpha)$ by the fractional part of a positive multiple of $\alpha$ is $k \beta \bmod \alpha$.

The first negative multiple of $\alpha$ for which the fractional part is in $(0, \alpha)$ is $-n_{1}(\alpha) \alpha$, giving the value $\left\{-n_{1}(\alpha) \alpha\right\}=1-\left\{n_{1}(\alpha) \alpha\right\}=1-n_{1}(\alpha) \alpha=\alpha-\beta$. Each next hit in $(0, \alpha)$ is shifted $\beta$ to the left until $\alpha-k \beta$ dives under 0 . In that case we leave $(0, \alpha)$, but the previous hit was $\alpha-k \beta+\alpha$, which is 
in $(0, \alpha)$. Hence, the $k$ th hit in $(0, \alpha)$ by the fractional part of a negative multiple of $\alpha$ is $\alpha-(k \beta \bmod \alpha)$.

By noting that the hits by fractional parts of positive and negative multiples of $\alpha$ are alternating we see that in $(0, \alpha)$ we get the following sequence of hits:

$$
\begin{array}{r}
\alpha-(\beta \bmod \alpha), \beta \bmod \alpha, \alpha-(2 \beta \bmod \alpha), 2 \beta \bmod \alpha, \\
\alpha-(3 \beta \bmod \alpha), 3 \beta \bmod \alpha, \ldots .
\end{array}
$$

By multiplying each term by $1 / \alpha$ we get

$$
\begin{aligned}
1-\left(\frac{\beta}{\alpha} \bmod 1\right), \frac{\beta}{\alpha} \bmod 1,1- & \left(\frac{2 \beta}{\alpha} \bmod 1\right), \frac{2 \beta}{\alpha} \bmod 1, \\
& 1-\left(\frac{3 \beta}{\alpha} \bmod 1\right), \frac{3 \beta}{\alpha} \bmod 1, \ldots
\end{aligned}
$$

By defining $\tilde{\alpha}:=1-\beta / \alpha$, we can rewrite this as

$$
\{\tilde{\alpha}\},\{-\tilde{\alpha}\},\{2 \tilde{\alpha}\},\{-2 \tilde{\alpha}\},\{3 \tilde{\alpha}\},\{-3 \tilde{\alpha}\}, \ldots
$$

Hence, (7) is a scaled version of the sequence $S_{\alpha}$ (with a different irrational step size). This means that the partition of the subinterval $(0, \alpha)$ has exactly the same structure and properties as the partition of $(0,1)$. The same self-similarity holds for the subintervals $(\alpha, 2 \alpha), \ldots,\left(\left(n_{1 / 2}(\alpha)-1\right) \alpha\right.$, $\left.n_{1 / 2}(\alpha) \alpha\right)$, where $n_{1 / 2}(\alpha) \alpha$ is the largest multiple of $\alpha$ smaller than $1 / 2$. In these subintervals we get the same sequence (7), but now shifted by a multiple of $\alpha$ to the corresponding positions in the subinterval. By using symmetry we also find the same structure of lengths for the intervals $\left(1-n_{1 / 2}(\alpha) \alpha, 1-\left(n_{1 / 2}(\alpha)-1\right) \alpha\right), \ldots,(1-\alpha, 1)$. These intervals are mirror images of the subintervals $(0, \alpha), \ldots,\left(\left(n_{1 / 2}(\alpha)-1\right) \alpha, n_{1 / 2}(\alpha) \alpha\right)$.

Each cycle of $S_{\alpha}$ gives two hits in each of those intervals. We conclude that for all $k \in \mathbb{N}, k \geq 1$,

$$
\begin{aligned}
& (0, \alpha)\left(n_{k}(\alpha)\right) \simeq \cdots \simeq\left(\left(n_{1 / 2}(\alpha)-1\right) \alpha, n_{1 / 2}(\alpha) \alpha\right)\left(n_{k}(\alpha)\right) \\
\simeq & \left(1-n_{1 / 2}(\alpha) \alpha, 1-\left(n_{1 / 2}(\alpha)-1\right) \alpha\right)\left(n_{k}(\alpha)\right) \simeq \cdots \simeq(1-\alpha, 1)\left(n_{k}(\alpha)\right) .
\end{aligned}
$$

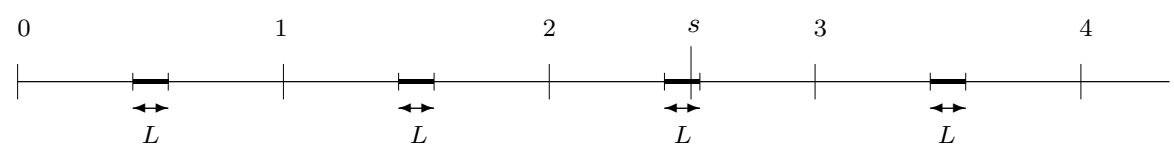

Fig. 2. Each interval between two integers consists of two parts of length $n_{1 / 2}(\alpha) \alpha$ and a part of length $L$ (thick). Fractional parts of numbers in the thick intervals are in $I_{m}$. The lengths of the bold parts to the left of $s$ add up to $\alpha$.

The only part which is not yet considered is the middle part of $(0,1)$ : the interval $\left(n_{1 / 2}(\alpha) \alpha, 1-n_{1 / 2}(\alpha) \alpha\right)$, which will be denoted by $I_{m}$ and its length by $L$. Denote the smallest positive multiple of $\alpha$ for which the fractional part is in $I_{m}$ by $s$, see Figure 2 . The Lebesgue measure of the set 


$$
\left\{x \in(0, s):\{x\} \notin I_{m}\right\}
$$

is a multiple of $n_{1 / 2}(\alpha) \alpha$. Hence the Lebesgue measure of the set

$$
\left\{x \in(0, s):\{x\} \in I_{m}\right\}
$$

must also be a multiple of $\alpha$. From the fact that $s$ is the smallest number for which the measure of this set is a multiple of $\alpha$ it follows that its measure is exactly $\alpha$. This implies that the first element of $S_{\alpha}$ which is in $I_{m}$ is given by

$$
\{s\}=n_{1 / 2}(\alpha) \alpha+(\alpha \bmod L) .
$$

For each next multiple of $\alpha$ giving a hit in $I_{m}$, a similar argument applies, but now the measure of the set (12) increases with $\alpha$ for each next hit. We conclude that the positive multiples of $\alpha$ give the following sequence of hits in $I_{m}$ :

$$
\left(n_{1 / 2}(\alpha) \alpha+(k \alpha \bmod L)\right)_{k=1}^{\infty} .
$$

By symmetry we see that by adding the negative multiples of $\alpha$ as well, we find the following sequence of hits in $I_{m}$ :

$$
\begin{aligned}
& n_{1 / 2}(\alpha) \alpha+(\alpha \bmod L), 1-n_{1 / 2}(\alpha) \alpha-(\alpha \bmod L), \\
& n_{1 / 2}(\alpha) \alpha+(2 \alpha \bmod L), 1-n_{1 / 2}(\alpha) \alpha-(2 \alpha \bmod L), \\
& n_{1 / 2}(\alpha) \alpha+(3 \alpha \bmod L), 1-n_{1 / 2}(\alpha) \alpha-(3 \alpha \bmod L), \ldots,
\end{aligned}
$$

where the alternating order follows from the fact that the successor of $\{k \alpha\}$ in $S_{\alpha}$ is $\{-k \alpha\}$.

Subtract $n_{1 / 2}(\alpha) \alpha$ to get

$$
\begin{aligned}
& \alpha \bmod L, L-(\alpha \bmod L), 2 \alpha \bmod L, \\
& \quad L-(2 \alpha \bmod L), 3 \alpha \bmod L, L-(3 \alpha \bmod L), \ldots .
\end{aligned}
$$

Multiplying by $1 / L$ yields

$$
\begin{aligned}
\frac{\alpha}{L} \bmod 1,1 & -\left(\frac{\alpha}{L} \bmod 1\right), \frac{2 \alpha}{L} \bmod 1, \\
1 & -\left(\frac{2 \alpha}{L} \bmod 1\right), \frac{3 \alpha}{L} \bmod 1,1-\left(\frac{3 \alpha}{L} \bmod 1\right), \ldots
\end{aligned}
$$

This is exactly $S_{\alpha}$, with step size $\alpha / L$. It follows that (15) is a scaled and translated version of the sequence $S_{\alpha}$ with a different step size.

The next step is to find the relation between the behavior of the partition process in $I_{m}$ and its complement. The intervals $(0, L)$ and $I_{m}$ have the same length (by definition of $L$ ) and the distance between their left endpoints is a multiple of $\alpha$. From this we can conclude that in each cycle a value $x \in(0, L)$ is hit if and only if in the same cycle the point $x+n_{1 / 2}(\alpha)$ is hit in $I_{m}$. This reasoning is also valid when $(0, L)$ and $I_{m}$ are not disjoint (which is possible 
when $L>\alpha)$. By noting that $(0, L)\left(n_{1}(\alpha)\right) \simeq I_{m}\left(n_{1}(\alpha)\right)$ and using induction on $k$ it follows that for all $k \in \mathbb{N}, k \geq 1$ :

$$
(0, L)\left(n_{k}(\alpha)\right) \simeq I_{m}\left(n_{k}(\alpha)\right) .
$$

In words: after each complete cycle the two intervals $(0, L)$ and $I_{m}$ are partitioned in an equivalent way in the sense of Definition 2.

Proof of Theorem 1. We use induction on the cycle number $k$. Note that if the theorem holds for $n$, then to go to $n+1$ it suffices to check the second assertion of the theorem. We can see this as follows. If we had three lengths, then one of the longest subintervals is divided into two existing lengths, so we get nothing new. If we had two lengths, then we get one new length, being the difference of the two existing lengths. These remarks show that the "at most three" part of the first assertion and the requirement $l_{1}=l_{2}+l_{3}$ in the case of three lengths are not violated. The "at least two" part of the first assertion of the theorem follows from the irrationality of $\alpha$. If only one length is remaining, the interval $[0,1]$ must be divided into equal parts. But in this case $\alpha$ would be a rational number.

STEP 1. The first step in our induction argument is to show that during the first cycle (containing the first $2 n_{1}(\alpha)$ terms of $S_{\alpha}$ ) always one of the longest subintervals is divided into two intervals of which one has the second length occurring before the division. The first number in the sequence $S_{\alpha}$ is $\{\alpha\}$, so after adding this first number the interval $(0,1)$ is divided into two subintervals, one of length $\alpha$ and one of length $1-\alpha$, where the latter is the longest in view of our assumption that $\alpha<1 / 2$. So now this longest subinterval should be divided into a part of length $\alpha$ (the second length) and a remaining part. Because the second hit is $\{-\alpha\}=1-\alpha$, this is indeed the case. The process continues in the same way, each time reducing the length of the middle subinterval by $\alpha$, until the length of the middle subinterval becomes smaller than $\alpha$. Now this middle subinterval has length $\alpha-\beta$, by definition of $\beta$.

At this point we have two different lengths: $\alpha$ and $\alpha-\beta$. The situation is illustrated by Figure 3. We now distinguish two cases.

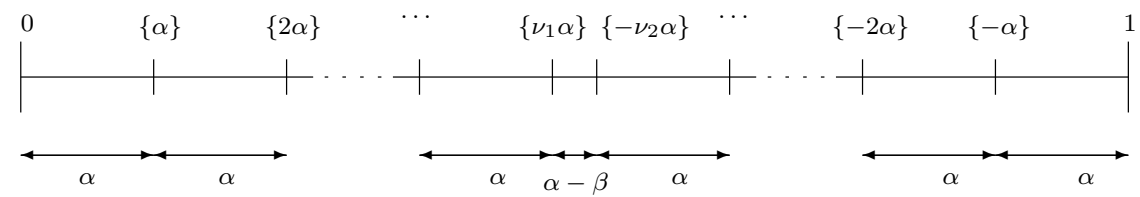

Fig. 3. Halfway the first cycle: either $\nu_{1}=\nu_{2}$ or $\nu_{1}=\nu_{2}+1$

If $\nu_{1}=\nu_{2}$, then the next hit will be $\left\{\left(\nu_{1}+1\right) \alpha\right\}$, dividing an interval of length $\alpha$ into a part of length $\alpha-\beta$ (which was the second length) and a part of length $\beta$ (a new length). Now we have three different lengths and the 
sum of the two smallest equals the largest, as required. The next hit now is $\left\{-\left(\nu_{1}+1\right) \alpha\right\}$ and again this divides an interval of length $\alpha$ into a part of length $\alpha-\beta$ and a part of length $\beta$. The partition process continues in this way as long as we are in the first cycle.

If $\nu_{1}=\nu_{2}+1$, then the next hit will be $\left\{-\nu_{1} \alpha\right\}$ and also in this case all intervals of length $\alpha$ will successively be divided into a part of length $\alpha-\beta$ and a part of length $\beta$.

Hence we conclude that the theorem is valid for the first cycle.

SteP 2. The next step in the induction argument is to show that if the theorem holds in the first $k$ cycles, then it also holds in the next cycle. To prove this we use the observations made before, which state that the behavior of the partition process in each of the intervals

$$
\begin{array}{r}
(0, \alpha), \ldots,\left(\left(n_{1 / 2}(\alpha)-1\right) \alpha, n_{1 / 2}(\alpha) \alpha\right),\left(n_{1 / 2}(\alpha) \alpha, 1-n_{1 / 2}(\alpha) \alpha\right), \\
\left(1-n_{1 / 2}(\alpha) \alpha, 1-\left(n_{1 / 2}(\alpha)-1\right) \alpha\right), \ldots,(1-\alpha, 1)
\end{array}
$$

has after rescaling the same properties as the behavior in the entire interval $(0,1)$. From now on we will call these intervals elementary intervals.

A crucial remark is that all boundaries (except 0 and 1 ) of the elementary intervals belong to the first cycle of $S_{\alpha}$. This implies that (at any point in one of the next cycles) the subintervals into which $(0,1)$ is divided can only intersect one of the elementary intervals. This guarantees that to find all lengths of subintervals in $(0,1)$, it suffices to find all lengths in the elementary intervals.

For the elementary intervals we introduce the following abbreviations:

$$
\begin{aligned}
I^{p} & :=((p-1) \alpha, p \alpha), \\
I^{-p} & :=(1-p \alpha, 1-(p-1) \alpha),
\end{aligned}
$$

where $1 \leq p \leq n_{1 / 2}(\alpha), p \in \mathbb{N}$. Recall that for the middle elementary interval we already introduced the symbol $I_{m}$. The sequence of hits in an elementary interval $I$ will be denoted by $S_{\alpha}^{I}$. For example, $S_{\alpha}^{I^{1}}$ is equal to the sequence (7). Because these sequences are scaled and translated versions of $S_{\alpha}$ (possibly with a different step size), we can also here introduce cycles. Every element of the $k$ th cycle of $S_{\alpha}$ is, for some $I$ and $l$, also an element of the $l$ th cycle of $S_{\alpha}^{I}$, where $l$ may be different from $k$. We are going to use these cycles later, but we do not need to specify them explicitly.

Induction hypothesis. Assume that for all $\alpha$ and some $k \geq 1$ the theorem holds as long as we are in one of the first $k$ cycles of $S_{\alpha}$.

Let $2 n_{k}(\alpha) \leq n<2 n_{k+1}(\alpha)$, implying that $S_{\alpha}(n+1)$ is an element of the $(k+1)$ th cycle of $S_{\alpha}$. Consider the partition of $(0,1)$ into subintervals by the first $n$ terms of $S_{\alpha}$. Denote the lengths of the subintervals by $l_{1}>l_{2}\left(>l_{3}\right)$. 
To prove that the theorem holds for the $(k+1)$ th cycle, it suffices to show that the following three requirements are satisfied:

REQUiREMENT 1. If $S_{\alpha}(n+1)$ is the very first hit in an elementary interval, then it splits a subinterval of length $l_{1}$ into subintervals of length $l_{2}$ and $l_{1}-l_{2}$.

REQUiREment 2. If $S_{\alpha}(n+1) \in I$, where $I$ is one of the elementary intervals, then $I$ contains a subinterval of length $l_{1}$ just before $S_{\alpha}(n+1)$ is added.

REQUIREMENT 3. If $S_{\alpha}(n+1)$ is not the very first hit in an elementary interval, denote the two largest lengths in this elementary interval by $\hat{l}_{1}>\hat{l}_{2}$. Then $S_{\alpha}(n+1)$ splits a subinterval of length $\hat{l}_{1}$ into subintervals of length $\hat{l}_{2}$ and $\hat{l}_{1}-\hat{l}_{2}$.

First we argue why it is sufficient that these three requirements hold and then we check each of them in the substeps below.

The idea of the proof is to use the self-similar structure by applying the induction hypothesis to the elementary intervals. The theorem only gives an assertion about the division into subintervals if we already have at least two lengths. Hence, our induction hypothesis makes no statement about the very first hit in an elementary interval. Therefore, in Substep 2.1 we start by checking that in each of the elementary intervals the partition process starts in the right way, as indicated by Requirement 1. Suppose $I$ is the elementary interval containing $S_{\alpha}(n+1)$. Denote the lengths occurring in $I$ just before adding $S_{\alpha}(n+1)$ by $\hat{l}_{1}>\hat{l}_{2}\left(>\hat{l}_{3}\right)$. Then the maximal length in $I$ should equal the maximal length in $[0,1]: \hat{l}_{1}=l_{1}$, which is Requirement 2 . Since all lengths in $I$ are also lengths in $[0,1]$, either $\hat{l}_{2}=l_{2}$ or $\hat{l}_{2}=l_{3}$. In both cases, splitting an interval of length $\hat{l}_{1}$ into two subintervals of lengths $\hat{l}_{2}$ and $\hat{l}_{1}-\hat{l}_{2}$ (as is demanded in Requirement 3) is the same as splitting an interval of length $l_{1}$ into two subintervals of lengths $l_{2}$ and $l_{1}-l_{2}$, since $l_{3}=l_{1}-l_{2}$. We conclude that these three requirements are sufficient to complete the proof. The induction hypothesis is only needed to prove Requirement 3.

SubsteP 2.1. All elementary intervals, except $I_{m}$ if $L<\alpha$, get at least one hit in the first cycle of $S_{\alpha}$. So here we have no problems, because we already checked that the theorem holds for the first cycle. Suppose that $L<\alpha$ and that $S_{\alpha}(n+1)$ is the first value we hit in $I_{m}$. Then $S_{\alpha}(n+1)$ can be written as $n_{1 / 2}(\alpha) \alpha+x$, where $x \in(0, L)$. In the same cycle the value $x$ was already hit in $(0, L)$. The hit $n_{1 / 2}(\alpha) \alpha+x$ splits $I_{m}$ in exactly the same way as $x$ has divided $(0, L)$. This means that two subintervals are generated with lengths already occurring before the division. The two new subintervals have lengths $l_{2}$ and $l_{3}=l_{1}-l_{2}$. 
Substep 2.2. After each complete cycle of $S_{\alpha}, I_{m}$ is partitioned in a symmetric way. This implies that the longest subinterval occurring in $I_{m}$ is a subinterval of $\left(n_{1 / 2}(\alpha) \alpha,\left(n_{1 / 2}(\alpha)+1\right) \alpha\right)$. From this observation, combined with (10) and (18), it follows that after each complete cycle all the intervals $I^{p}, I^{-p}$, where $1 \leq p \leq n_{1 / 2}(\alpha)$, contain a subinterval which has the maximal length. Now note that in each cycle the order in which the elementary intervals will get hits is as follows (writing $n_{1 / 2}(\alpha)$ as $n_{1 / 2}^{\alpha}$ for typographical reasons):

$$
\underbrace{I^{1}, I^{-1}, I^{2}, I^{-2}, \ldots, I^{n_{1 / 2}^{\alpha}}, I^{-n_{1 / 2}^{\alpha}}}_{\text {1st sequence }}, \underbrace{I_{m}, \ldots, I_{m}}_{\text {2nd sequence }}, \underbrace{I^{-n_{1 / 2}^{\alpha}, I^{n_{1 / 2}^{\alpha}}, \ldots, I^{-2}, I^{2}, I^{-1}, I^{1}}}_{\text {3rd sequence }},
$$

where the second sequence contains 0,2 or 4 elements. Observe that the equivalences

$$
\begin{aligned}
(0, \alpha) \simeq & \cdots \simeq\left(\left(n_{1 / 2}(\alpha)-1\right) \alpha, n_{1 / 2}(\alpha) \alpha\right) \\
& \simeq\left(1-n_{1 / 2}(\alpha) \alpha, 1-\left(n_{1 / 2}(\alpha)-1\right) \alpha\right) \simeq \cdots \simeq(1-\alpha, 1)
\end{aligned}
$$

hold after the first sequence and after the second sequence. At the start of the cycle, all elementary intervals in the first sequence contain a subinterval of the maximal length. It follows that Requirement 2 is satisfied if $S_{\alpha}(n+1)$ belongs to the first sequence.

Now let us first check the third sequence. After the third sequence of hits a cycle is completed, so then again all the intervals $I^{p}, I^{-p}$, where $1 \leq p \leq n_{1 / 2}(\alpha)$, contain a subinterval which has the maximal length. Just before the third sequence the maximal subinterval in each of these elementary intervals was certainly not smaller. Since (23) holds after the second sequence, those maximal subintervals all had the same length, which shows that Requirement 2 is satisfied if $S_{\alpha}(n+1)$ belongs to the third sequence.

The hits corresponding to the second sequence in (22) can only violate Requirement 2 if the last of these hits does so. This last hit gives a value in $\left(n_{1 / 2}(\alpha) \alpha,\left(n_{1 / 2}(\alpha)+1\right) \alpha\right)$. After the third sequence the $(k+1)$ th cycle is complete and hence we have the equivalence $(0, \alpha)\left(n_{k+1}(\alpha)\right) \simeq$ $\left(n_{1 / 2}(\alpha) \alpha,\left(n_{1 / 2}(\alpha)+1\right) \alpha\right)\left(n_{k+1}(\alpha)\right)$. The third sequence gives only one hit in $(0, \alpha)$. The distance between this hit and the last hit of the second sequence is $n_{1 / 2}(\alpha) \alpha$. It follows that the last hit of the second sequence splits an interval into two subintervals in exactly the same way as the third sequence does in $(0, \alpha)$. By equivalences and symmetry the same holds for the other elementary intervals. Hence, Requirement 2 is also satisfied if $S_{\alpha}(n+1)$ belongs to the second sequence.

SubSTEP 2.3. To check Requirement 3 we use our induction hypothesis. Suppose $S_{\alpha}(n+1)$ is a hit in $I$, where $I$ is one of the elementary intervals. If 
$S_{\alpha}(n+1)$ is an element of one of the first $k$ cycles of $S_{\alpha}^{I}$, then by our induction hypothesis it follows that $S_{\alpha}(n+1)$ divides an interval of length $\hat{l}_{1}$ into a part of length $\hat{l}_{2}$ and a part of length $\hat{l}_{1}-\hat{l}_{2}$, where $\hat{l}_{1}>\hat{l}_{2}\left(>\hat{l}_{3}\right)$ are the lengths of the subintervals in $I$, and we are ready.

Suppose that the elementary interval $I$ containing $S_{\alpha}(n+1)$ has length not larger than $\alpha$. Then each cycle of $S_{\alpha}$ gives at most 2 hits in $I$. After $k+1$ complete cycles of $S_{\alpha}$, we have recorded at most $2(k+1)$ values in $I$. After $k$ complete cycles of $S_{\alpha}^{I}$, we have at least $4 k$ hits in $I$. Since $4 k \geq 2(k+1)$, $S_{\alpha}(n+1)$ belongs to one of the first $k$ cycles of $S_{\alpha}^{I}$ and Requirement 3 is satisfied because of the induction hypothesis.

Suppose that $S_{\alpha}(n+1) \in I_{m}$ and $L>\alpha$ (this is the only case where the argument from the previous paragraph fails). Note that from the definitions it follows that $L=2 \alpha-\beta$. In the first cycle of $S_{\alpha}$ we get 2 hits in $I_{m}$. Each next cycle of $S_{\alpha}$ gives either 2 or 4 hits in $I_{m}$. After $k+1$ complete cycles of $S_{\alpha}$, we have recorded at most $4 k+2$ values in $I_{m}$. After $k$ complete cycles of $S_{\alpha}^{I_{m}}$, we have at least $4 k$ hits in $I_{m}$. It follows that if the $(k+1)$ th cycle of $S_{\alpha}$ gives two hits in $I_{m}$, then $S_{\alpha}(n+1)$ belongs to one of the first $k$ cycles of $S_{\alpha}^{I_{m}}$ and again we use the induction hypothesis to conclude that Requirement 3 is satisfied.

If the $(k+1)$ th cycle of $S_{\alpha}$ gives four hits in $I_{m}$, denote the last two hits by $x_{1}$ and $x_{2}$. We can only have a problem when $S_{\alpha}(n+1)$ is equal to $x_{1}$ or $x_{2}$, since otherwise $S_{\alpha}(n+1)$ belongs to one of the first $k$ cycles of $S_{\alpha}^{I_{m}}$. So we check if $x_{1}$ and $x_{2}$ split an interval according to Requirement 3 . Note that $x_{1}$ and $x_{2}$ are in $\left(\left(n_{1 / 2}(\alpha)+1\right) \alpha, 1-n_{1 / 2}(\alpha) \alpha\right)$ and $\left(n_{1 / 2}(\alpha) \alpha, 1-\left(n_{1 / 2}(\alpha)+1\right) \alpha\right)$ respectively. These intervals both have length $L-\alpha=\alpha-\beta$. The distance between $x_{2}$ and the next hit $x$ in $(0, \alpha-\beta)$ is a multiple of $\alpha$ and by (18) we know that at the moment that $x$ is reached in $S_{\alpha}$, we have $\left(n_{1 / 2}(\alpha) \alpha, 1-\right.$ $\left.\left(n_{1 / 2}(\alpha)+1\right) \alpha\right) \simeq(0, \alpha-\beta)$. Hence $x_{2}$ splits $\left(n_{1 / 2}(\alpha) \alpha, 1-\left(n_{1 / 2}(\alpha)+1\right) \alpha\right)$ in exactly the same way as $x$ splits $(0, \alpha-\beta)$. Since $x$ belongs to one of the first $k$ cycles of $S_{\alpha}^{I^{1}}$, we already know that $x$ gives the right splitting. Therefore Requirement 3 is satisfied if $x_{2}=S_{\alpha}(n+1)$. Using symmetry we see that Requirement 3 is also satisfied when $x_{1}=S_{\alpha}(n+1)$, which completes the proof.

3. A Four Gap Theorem. We are now in a position to prove our main theorem, the "Four Gap Theorem".

Theorem 2 (The Four Gap Theorem). Let $\alpha \in \mathbb{R} \backslash \mathbb{Q}$ and $n \in \mathbb{N}$. Let $\|x\|$ denote the distance from $x$ to the nearest integer. The numbers

$$
\|\alpha\|,\|2 \alpha\|,\|3 \alpha\|, \ldots,\|n \alpha\|
$$

divide the interval $[0,1 / 2]$ into subintervals of at least two and at most four 
different lengths. For these lengths the following assertions hold:

1. The rightmost length, denoted by $l_{r}$, is unique.

2. There are two different lengths if and only if $n\|\alpha\|<1 / 2$.

3. If we have three different lengths, denote the two lengths not equal to $l_{r}$ by $l_{1}>l_{2}$. Then exactly one of the following four equalities holds: $2 l_{r}=l_{1}, 2 l_{r}=l_{2}, 2 l_{r}+l_{2}=l_{1}$ or $l_{1}+l_{2}=2 l_{r}\left({ }^{1}\right)$.

4. If we have four different lengths, denote the three lengths not equal to $l_{r}$ by $l_{1}>l_{2}>l_{3}$. Then $l_{1}=l_{2}+l_{3}$ and one of these lengths is equal to twice $l_{r}$.

Proof. It is not possible to have only one length occurring, since $\alpha$ is irrational. Without loss of generality we assume that $\alpha \in[0,1 / 2]$.

Observe that $\min \{\{x\},\{-x\}\} \in[0,1 / 2]$. So if we look at the sequence

$$
\min \{\{\alpha\},\{-\alpha\}\}, \min \{\{2 \alpha\},\{-2 \alpha\}\}, \min \{\{3 \alpha\},\{-3 \alpha\}\}, \ldots,
$$

we get a subsequence of the sequence $S_{\alpha}$. A term of $S_{\alpha}$ is a term of the sequence (25) if and only if it is in $[0,1 / 2]$. Consequently, by Theorem 1 , the first $n$ terms of the sequence (25) divide the interval $[0,1 / 2]$ into subintervals of at least two and at most four different lengths. We possibly get a fourth length because the partition of $[0,1]$ (which gave three lengths) is now truncated at $1 / 2$. Since

$$
\min \{\{n \alpha\},\{-n \alpha\}\}=\|n \alpha\|,
$$

the numbers in (24) divide $[0,1 / 2]$ into subintervals of at least two and at most four different lengths.

We now turn our attention to the four assertions about the lengths. If the rightmost length is not unique, then there exist integers $0 \leq k, l, m \leq n$, with $l \neq m$, such that

$$
1 / 2-\|k \alpha\|=\|l \alpha\|-\|m \alpha\|,
$$

which implies that $1 / 2$ is the sum of a multiple of $\alpha$ and an integer, contradicting the irrationality of $\alpha$. Hence, the rightmost length $l_{r}$ is unique.

If $n\|\alpha\|<1 / 2$, then the only lengths are $\|\alpha\|$ and $l_{r}$, so we only have two different lengths. For the opposite implication, assume that there are only two different lengths. The leftmost interval has length $\min _{1 \leq k \leq n}\|k \alpha\|$. It follows that the numbers $\|\alpha\|, \ldots,\|n \alpha\|$ are all multiples of $\min _{1 \leq k \leq n}\|k \alpha\|$. From the irrationality of $\alpha$ we conclude that $\min _{1 \leq k \leq n}\|k \alpha\|=\|\alpha\|$ and $\|n \alpha\|=n\|\alpha\|$, which is only possible if $n\|\alpha\|<1 / 2$.

Consider the partition of $[0,1]$ by the numbers

$$
\{\alpha\},\{-\alpha\},\{2 \alpha\},\{-2 \alpha\}, \ldots,\{n \alpha\},\{-n \alpha\} .
$$

$\left({ }^{1}\right)$ For all four possibilities we found an example. 
This partition is symmetric with respect to $1 / 2$. The subintervals into which $[0,1]$ is divided by these numbers have either two or three different lengths, according to Theorem 1 . We check what happens in both cases. If we have two different lengths and after truncating the partition of $[0,1]$ at $1 / 2$ have three different lengths, then either $2 l_{r}=l_{1}$ or $2 l_{r}=l_{2}$. If we have three different lengths and after truncating the partition at $1 / 2$ again have three different lengths, then either $2 l_{r}+l_{2}=l_{1}$ or $l_{1}+l_{2}=2 l_{r}$.

The last assertion of the Four Gap Theorem follows immediately from Theorem 1 and the observations made before.

Acknowledgements. The author thanks Cor Kraaikamp and Rob Tijdeman for their useful comments.

\section{References}

[1] T. van Ravenstein, The three gap theorem (Steinhaus conjecture), J. Austral. Math. Soc. Ser. A 45 (1988), 360-370.

[2] N. B. Slater, Gaps and steps for the sequence $n \theta \bmod 1$, Proc. Cambridge Philos. Soc. 63 (1967), 1115-1123.

[3] V. T. Sós, On the distribution mod 1 of the sequence na, Ann. Univ. Sci. Budapest. Eötvös Sect. Math. 1 (1958), 127-134.

[4] J. Surányi, Über die Anordnung der Vielfachen einer reellen Zahl mod 1, ibid., 107111.

[5] S. Świerczkowski, On successive settings of an arc on the circumference of a circle, Fund. Math. 46 (1958), 187-189.

Section Probability Theory

TU Delft, EWI (DIAM)

Mekelweg 4

2628 CD Delft, The Netherlands

E-mail: henkdon@gmail.com

Received on 22.8.2008

and in revised form on 18.3.2009 